\title{
New Results in Feedback Control of Unsupported Standing in Paraplegia
}

\author{
Henrik Gollee, Member, IEEE, Ken J. Hunt, Member, IEEE, and Duncan E. Wood
}

\begin{abstract}
The aim of this study was to implement a new approach to feedback control of unsupported standing and to evaluate it in tests with an intact and a paraplegic subject. In our setup, all joints above the ankles are braced and stabilizing torque at the ankle is generated by electrical stimulation of the plantarflexor muscles. A previous study showed that short periods of unsupported standing with a paraplegic subject could be achieved. In order to improve consistency and reliability and to prolong the duration of standing, we have implemented several modifications to the control strategy. These include a simplified control structure and a different controller design method. While the reliability of standing is mainly limited by the muscle characteristics such as reduced strength and progressive fatigue, the results presented here show that the new strategy allows much longer periods (up to several minutes) of unsupported standing in paraplegia.
\end{abstract}

Index Terms-Balancing, feedback control, functional electrical stimulation (FES), paraplegia.

\section{INTRODUCTION}

$\mathbf{F}$ OR MUSCLE which has lost voluntary central nervous control, artificial electrical stimulation can be used to provide muscular contraction and functionally useful movement. This is generally referred to as functional electrical stimulation (FES) [1], [2]. The technique can be used with spinal-cord injured subjects to restore some normal motor activities to the paralyzed muscles to provide functions such as standing up [3], [4], standing [5], [6], and stepping [7].

We are investigating the use of feedback control systems which enable paraplegics to stand without the support of their hands and arms - we call this unsupported standing. FES of the plantarflexor muscles is used to generate a moment at the ankle joints which stabilizes the upright posture. In our experimental setup, all joints above the ankle are locked using a special body brace, allowing us to isolate the effects of the artificial control system from the remaining motor control actions of the intact upper body. While we believe that functional systems must integrate the natural and artificial controllers, our experimental setup allows us to study the potential benefits and fundamental limitations of the artificial system.

The simulation of unsupported standing has been studied by Khang and Zajac [8], who described the body as a multilink system. In a simplified setup, the body can be interpreted as a single link inverted pendulum, assuming that the knee and hip

Manuscript received May 14, 2002; revised January 13, 2003.

H. Gollee and K. J. Hunt are with the Center for Rehabilitation Engineering, Department of Mechanical Engineering, University of Glasgow, Glasgow G12 8QQ, Scotland, U.K. (e-mail: H.Gollee@eng.gla.ac.uk).

D. E. Wood is with the Department of Medical Physics and Biomedical Engineering, Salisbury District Hospital, Salisbury SP2 8BJ, U.K.

Digital Object Identifier 10.1109/TNSRE.2003.822765 joints are locked [9]. This system can be stabilized by generating a corresponding moment at the ankle joint, using an appropriate feedback control scheme [10]. In a previous experimental study [11], [12], [5], a nested control structure was proposed in which an inner loop controls the moment at the ankle, while an outer loop regulates the inclination angle of the body. It was found that this approach is feasible; a neurologically intact subject can be stabilized for long periods of time, and the controller is able to maintain stability in the face of significant disturbances. The study also showed that short periods of unsupported standing could be achieved with a paraplegic subject [5]. We concluded that the principal limitations to the approach included limited muscle strength, rapid fatigue, and significant spasticity of the paralyzed muscles. While these limitations are dependent on the condition of the particular subject in question, the underlying parameterization and design of the artificial controller has a crucial effect on the length of time during which successful standing is achieved. Thus, it is required for the controller to be robust enough to deal with sources of uncertainty and disturbance such as fatigue and spasticity, while at the same time it must maintain upright postural stability as safely and dependably as possible. Furthermore, it is desirable that the controller parameters are easy and quick to tune.

We have investigated a number of modifications to the original control structure proposed in [5]. The details of the new control approach are given in [13]. Modifications include changes to the control algorithm and control structure, with the aim being to improve the consistency and reliability of unsupported standing. Results with neurologically intact subjects [14] show that our modified control strategy performs reliably. In this paper, these initial results are discussed together with results from a new study with a paraplegic subject which show that significant periods of unsupported standing can be achieved.

\section{METHODS}

\section{A. Apparatus}

To perform dynamic tests of unsupported standing, an apparatus called the "Wobbler" was constructed. Full details of the construction and functionality of the Wobbler are given elsewhere [12].

While standing in the Wobbler apparatus (see Fig. 1), the subject is supported by a body brace which extends from above the ankle to the neck and locks all joints in this region. The feet are locked in boxes which allows measurement of the moments generated at each ankle. The body is attached to four safety ropes at shoulder level which prevent the subject from falling. When these ropes are taut, the body is fixed in an upright posture. For 


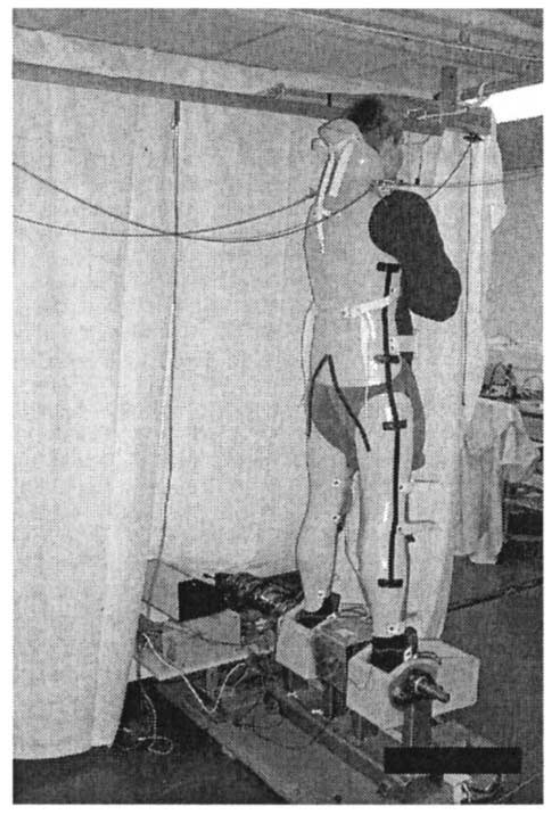

Fig. 1. Subject in the Wobbler apparatus.

standing experiments, the ropes are slackened to allow the body to move back and forth in the sagittal plane. The angle of inclination of the body is measured by a string attached to the back of the body brace which is wound around a pulley attached to a potentiometer placed well behind the subject.

\section{B. Modeling and Control Strategy}

A nested control structure for unsupported standing is shown in Figs. 2 and 3. An inner-loop controller $C_{m}$ regulates the total ankle moment $m$ by applying a stimulation pulsewidth $p$. The outer-loop controller $C_{\theta}$ regulates the body inclination angle $\theta$ by providing a desired (reference) moment $m_{\text {ref }}$ for the inner loop. The reference angle is $\theta_{\text {ref }}$.

1) Modeling: The design of the inner-loop moment controller $C_{m}$ is based on an empirical linear-dynamic model of the muscle response from stimulation $p$ to moment $m$. This model is derived from a series of identification tests which are carried out at the start of each experimental session. A series of stimulation signals in pseudorandom binary sequence (PRBS) form is applied in open loop at a range of mean levels and the total moment generated is recorded. This allows a family of linear, second-order models to be identified using a linear least-squares approach [15]. Usually the model with the highest static gain is chosen as the nominal model $G_{m}$ and used to design a single linear controller $C_{m}$, which is then employed over the entire operating range for the muscles. Note that due to the small-signal approach to system identification (ie., the identification of a system in a small region around nominal a operating point) we are able to neglect the nonlinear recruitment curve of the muscle which simplifies the identification procedure.

The design of the outer-loop position controller $C_{\theta}$ is based on a model comprising a linearized model of the body dynamics and a model of the closed inner control loop. The latter can be described as the control loop consisting the moment controller

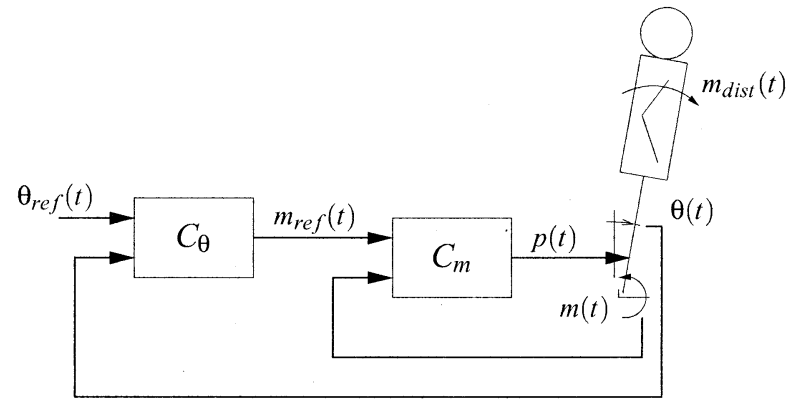

Fig. 2. Nested controller: General structure.

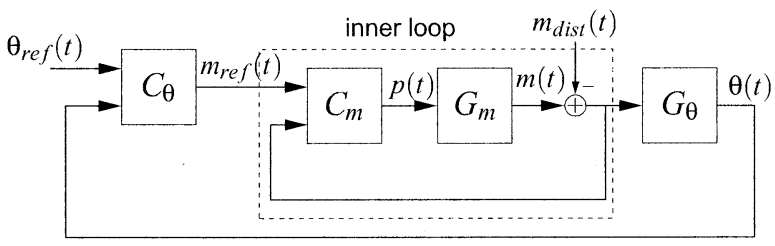

Fig. 3. Nested controller: Schematic structure.

$C_{m}$ and the linear model used to design this controller $G_{m}$. The body dynamics can be described as an inverted pendulum

$$
J \ddot{\theta}(t)=\tilde{m} g l \theta(t)-m(t)+m_{\mathrm{dist}}(t) .
$$

Here, $\theta$ is the inclination angle and $\ddot{\theta}$ describes its second derivative with respect to time. We have assumed that $\sin \theta=\theta$ for small angles; $m(t)$ is the total ankle moment, and $m_{\text {dist }}(t)$ describes an external disturbance moment. The inverted pendulum (i.e., the body moving around the ankle joint) is characterized by its moment of inertia $J$, its mass $\tilde{m}$, and the distance of the center of mass from the ankle $l$. The constant $g$ is the gravitational acceleration. The parameters $J, \tilde{m}$, and $l$ are determined once for each subject using the procedure described in [12] and assumed to be time-invariant.

When (1) is rewritten using the Laplace transform complex variable $s$, the continuous time transfer function for the body $G_{\theta}(s)$ can be derived as

$$
G_{\theta}(s)=\frac{\Theta(s)}{M(s)}=\frac{\frac{-1}{J}}{s^{2}-\frac{\tilde{m} g l}{J}}
$$

where $\Theta(s)$ is the Laplace transform of the angle $\theta(t)$, and $M(s)$ is the Laplace transform of the total moment at the ankle $m(t)-$ $m_{\text {dist }}(t)$.

The disturbance moment $m_{\text {dist }}$ cannot generally be measured directly. It is, however, possible to estimate it from measurements of $m(t)$ and $\theta(t)$. From (1), it follows that the disturbance moment is

$$
m_{\text {dist }}(t)=m(t)+J \ddot{\theta}(t)-\tilde{m} g l \theta(t) .
$$

2) Controller Design: Our previous control approach [5] was modified in two significant ways. In our previous study, the total moment requested by the outer loop was distributed equally as a reference moment to separate inner-loop controllers for the left and for the right muscles. Thus, if one side fatigues, the stimulation to this side would be increased, though not 
necessarily with an increase in moment as the stimulation saturates. However, the other nonfatigued side might still have the ability to generate additional moment. In our present approach, the same stimulation is applied to both muscles in such a way that the total moment generated by both sides follows the moment requested from the outer loop. Thus, the difference in the strength of the muscles is compensated for. At the same time, the structure of the inner-loop controller is simplified since only a single controller is now required for the inner-loop which regulates the total ankle moment. A side-effect of this modification is that the moment generated can now be different at each ankle, depending on the muscle strength. Although this moment difference could potentially cause a movement in the frontal plane this was not observed in our experiments.

The second important difference to the previous approach is the controller design method. In [5], a linear quadratic Gaussian (LQG) design was used which aims at minimizing the control error and the control effort according to a given criterion. While this approach provides a straightforward way of tuning the closed-loop characteristic for a given plant by selecting the parameters for the criterion accordingly, the characteristics of the closed loop are generally different for different plant characteristics. In order to obtain consistent closed loop performance it is, therefore, necessary to adjust the control design parameters whenever the plant characteristics have changed, e.g., for experiments with the same subject on different days.

In our present study, we employ a standard pole-placement design approach for both the inner and the outer loop controllers (see [13] for full details and [16] for a description of the pole-placement design approach). Here, the desired closed loop characteristics are defined by setting a rise-time and damping factor in the time-domain [17]. Thus, the closed-loop characteristics can be specified directly, and the same nominal closed-loop characteristics can be achieved with unmodified design parameters for plants with different characteristics (e.g., for experiments on different days). As a result, tuning of the controller becomes easier and more consistent for varying experimental conditions, and often the design parameters do not need to be modified at all. Integral action, which is required to ensure that the error between the reference value and the measured value approaches zero in the presence of constant disturbances or modeling errors (such as fatigue), is now only included in the design of the outer loop but not in the inner loop. Note, that this leads generally to an offset between the reference moment $m_{\text {ref }}$ and the measured moment $m$. This is, however, compensated in the outer loop by adjusting $m_{\text {ref }}$ accordingly.

A different controller design approach which was also investigated in this study, is aimed at taking explicitly account of the parameter variations (i.e., uncertainty) of the system by employing a robust control approach based on the $\mathrm{H}_{\infty}$ method. While this results in a controller with given stability and performance robustness with respect to the prescribed plant uncertainty, tuning of the controller design parameters is more complex, requiring the selection of a number of weighting functions. Full details and results for this approach are reported elsewhere [18], [19].

\section{Subjects}

Two subjects participated in this study. Subject SI was a fit and healthy 29-year-old male with no neurological deficit. His body parameters are $J=85 \mathrm{Nms}^{2}, \tilde{m}=75 \mathrm{~kg}$, and $l=1 \mathrm{~m}$.

Subject SP was a 44-year-old male, and a T7/8 complete-lesion paraplegic. At the time of the study, he was four years post-injury. Muscle training involved alternate stimulation of bilateral plantarflexors as one group and bilateral dorsiflexors in the second group. Stimulation parameters were set for near maximal contraction- $300 \mu \mathrm{s} ; 20 \mathrm{~Hz}$; anterior tibialis m: left 30 , right $40 \mathrm{~mA}$; gastrocnemius $\mathrm{m}$ : left 70, right $90 \mathrm{~mA}$. Profile of the stimulation sequence was a $50 \%$ duty cycle: 12 s ON (including 4-s ramp up and 4-s ramp down) and 12 s OFF. Initially retraining was for $30 \mathrm{~min}$ per day and then increased to $1 \mathrm{~h}$ per day after four weeks. The subject's muscles were trained for 12 weeks prior to the first experimental session, and regular training continued throughout the study which lasted for approximately seven months. His body parameters were determined to be $J=110 \mathrm{Nms}^{2}, \tilde{m}=90 \mathrm{~kg}$, and $l=1 \mathrm{~m}$.

During the experimental sessions, the plantarflexors are stimulated by pairs of self-adhesive electrodes (diameter $50 \mathrm{~mm}$ for subject SI, $75 \mathrm{~mm}$ for subject $\mathrm{SP}$ ) which are placed over the midline of the plantarflexor muscle group. The stimulator [20] uses a predefined current and produces monophasic stimulation pulses with a variable pulsewidth (range of 0-500 $\mu$ s for subject SI, 0-800 $\mu$ s for subject SP) with a frequency of $20 \mathrm{~Hz}$. A custom-made body brace (padded vacuum-formed polythene shells, reinforced and joined by steel strips) was used to lock all joints above the ankle.

\section{Experimental Protocol}

During each experimental session, a number of tests were carried out. For the first three tests described in the following ( $A$, $P R B S$, and Moment), the subject is standing upright while the safety ropes are taut. Thus, the experimental setup is approximately isometric since the body cannot move. For test Standing, the safety ropes are slackened and the body can move back and forth.

1) Test A: The aim of this test is to set the stimulation current and to ensure that the stimulation electrodes are placed correctly and have not become unattached during transition into the body brace and the Wobbler apparatus. For a constant stimulation current, the stimulation pulsewidth is ramped up from 0 to the maximal pulsewidth over a period of $5 \mathrm{~s}$ and the generated moment is recorded. The test is repeated for different values of the current until the generated moment covers the entire range up to saturation. If necessary, the positions of the electrodes are adjusted to obtain maximal muscle contraction. This test typically takes approximately 1 min.

2) Test PRBS: This test is used to collect data for the identification of the empirical muscle model. The muscles are stimulated by a range of PRBS signals with fixed amplitude and varying mean levels, and the total moment is recorded. These data are then used as described in Section II-B1. Typically, it takes 2 min to perform this test. 
3) Test Moment: This test is used to verify the inner-loop moment controller. After the controller is designed based on the model identified from the data collected in test $P R B S$, the controller is tested by applying a step-wise changing reference moment $m_{\text {ref }}$ with different step sizes and at various levels. It takes typically $2-3$ min to perform this test.

4) Test Standing: For this test, the outer-loop controller $C_{\theta}$ is designed and the complete structure shown in Fig. 3 is implemented. The controller is tested for different conditions:

a) quiet standing: keeping the reference angle constant;

b) external disturbances: while the reference angle is constant, various disturbances are applied (pulling/pushing of the subject, the subject stretches his arm with a weight, etc.);

c) changing reference: step-wise varying reference angle.

For the paralyzed subject, tests $A, P R B S$, and Moment were done as quickly as possible to avoid unnecessary fatigue prior to the main test Standing.

\section{RESULTS}

\section{A. Subject SI-Neurologically Intact}

For subject SI, the stimulation current was typically set to $60 \mathrm{~mA}$ following Test A (see Section II-D). Input-output data recorded from the open-loop identification test (Test PRBS) are shown in Fig. 4. The top graph shows the stimulation pulsewidth $p$ for three different mean levels. The corresponding total ankle moments $m$ are depicted in the bottom plot. The parameters of the second order linear models which were identified from these three data sets are summarized in Table I. The transfer functions are given in the discrete-time $z$-domain. It can be seen that the gain, the damping ratio and the time-constants change with varying stimulation levels [21]. The model identified for a mean pulsewidth of $200 \mu \mathrm{s}$ has the highest gain and was, therefore, selected as the nominal model as it ensures robust stability of the closed loop.

The inner and outer control loops are designed using the poleplacement approach, with a closed-loop rise-time of $0.2 \mathrm{~s}$ and an observer rise-time of $0.1 \mathrm{~s}$ for the inner loop, and a closed-loop rise-time of $1 \mathrm{~s}$ and an observer rise-time of $0.7 \mathrm{~s}$ for the outer loop (see [13] for details).

Typical standing results are shown in Fig. 5. Here, the top plot shows the stimulation pulsewidth $p$, the second plot depicts the total ankle moments (both reference $m_{\text {ref }}$ and measured $m$ ) and the third plot shows the body angle (both reference $\theta_{\text {ref }}$ and measured angle, $\theta$ ). The bottom plot shows the disturbance moment, $m_{\text {dist }}$, which was estimated using (3). In this test, the reference angle changes at 10, 20,30, and $40 \mathrm{~s}$. It can be seen that the actual angle follows the reference very accurately and within the given design characteristics. Additionally, disturbances of approximately $20 \mathrm{Nm}$ are applied at 25 (pulling the subject forward), 35 (pushing the subject backward), and at $45 \mathrm{~s}$ (again, pulling forward). These disturbances are rejected easily.
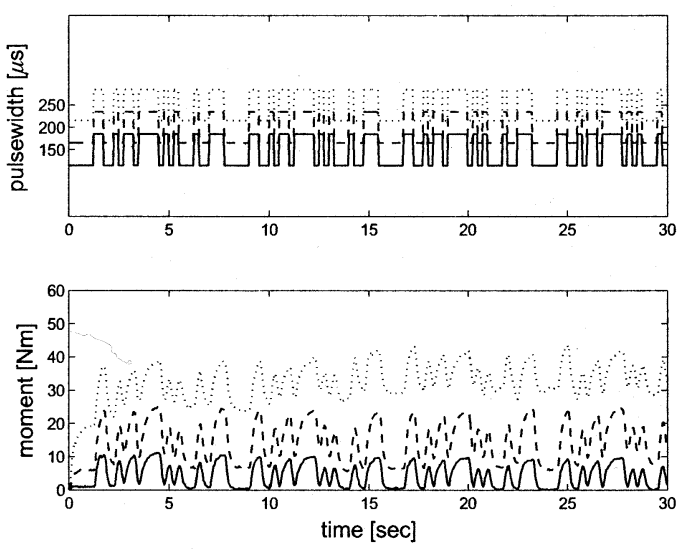

Fig. 4. Open-loop identification data, subject SI. Corresponding data and models are shown in the same line-style.

TABLE I

IDENTIFICATION RESULTS, SUBJECT SI

\begin{tabular}{c|c|c|c|c|c}
$\begin{array}{c}\text { mean } \\
\text { pulsewidth } \\
{[\mu \mathrm{s}]}\end{array}$ & $\begin{array}{c}\text { transfer } \\
\text { function } \\
G_{m}(z)\end{array}$ & poles & DC gain & $\begin{array}{c}\text { damping } \\
\text { ratio } \\
\xi\end{array}$ & $\begin{array}{c}\text { natural } \\
\text { frequencies } \\
\omega_{n n}[\mathrm{rad} / \mathrm{s}]\end{array}$ \\
\hline $150 \mu \mathrm{s}$ & $\frac{0.019 z}{z^{2}-1.33 z+0.49}$ & $0.67 \pm 0.21 i$ & 0.12 & 0.76 & 9.44 \\
$\mathbf{2 0 0} \mu \mathrm{s}$ & $\frac{\mathbf{0 . 0 2 7 \mathbf { z }}}{\mathbf{z}^{2}-\mathbf{1 . 4 1 2}+\mathbf{0 . 5 3}}$ & $\mathbf{0 . 7 1} \pm \mathbf{0 . 1 9 i}$ & $\mathbf{0 . 2 2}$ & $\mathbf{0 . 7 7}$ & $\mathbf{8 . 1 7}$ \\
$250 \mu \mathrm{s}$ & $\frac{0.021 z}{z^{2}-1.43 z+0.54}$ & $0.71 \pm 0.17 i$ & 0.19 & 0.80 & 7.75
\end{tabular}
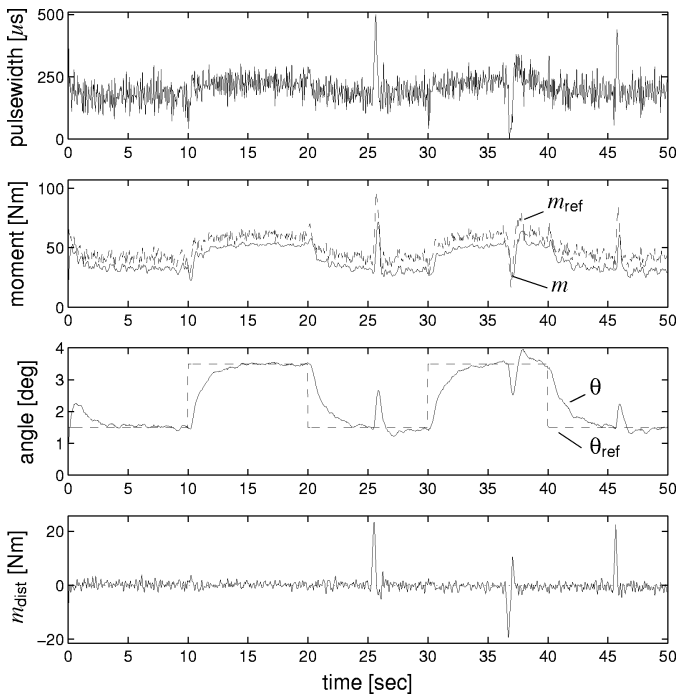

Fig. 5. Standing results, subject SI. Solid lines indicate measured values, dashed lines are reference values.

\section{B. Subject SP-Paraplegic}

For subject SP, the stimulation current was usually set to $120 \mathrm{~mA}$, following Test A (see Section II-D). Fig. 6 shows input-output data recorded during Test PRBS. The top graph shows the PRBS-like pulsewidth $p$ for three mean levels, while the bottom graph depicts the generated total ankle moments $m$. The results of the identification of the three corresponding linear second order models are summarized in Table II. The transfer functions are given in the discrete-time $z$-domain. 

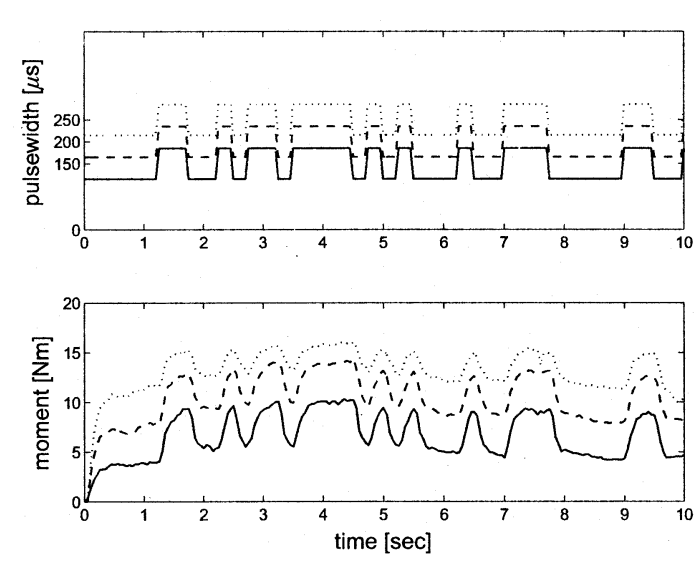

Fig. 6. Open-loop identification data, subject SP. Corresponding data and models are shown in the same line-style.

TABLE II

IDENTIFICATION RESULTS, SUBJECT SP

\begin{tabular}{c|c|c|c|c|c}
\hline $\begin{array}{c}\text { mean } \\
\text { pulsewidth } \\
{[\mu \mathrm{s}]}\end{array}$ & $\begin{array}{c}\text { transfer } \\
\text { function } \\
G_{m}(z)\end{array}$ & poles & DC gain & $\begin{array}{c}\text { damping } \\
\text { ratio } \\
\xi\end{array}$ & $\begin{array}{c}\text { natural } \\
\text { frequencies } \\
\omega_{n}[\text { rad } / \mathrm{s}]\end{array}$ \\
\hline $150 \mu \mathrm{s}$ & $\frac{0.018 z}{z^{2}-1.01 z+0.26}$ & $0.51 \pm 0.064 i$ & 0.070 & 0.98 & 13.7 \\
$\mathbf{2 0 0 \mu \mathrm { s }}$ & $\frac{\mathbf{0 . 0 1 5 z}}{\mathbf{z}^{2}-1.09 \mathbf{0}+\mathbf{0 . 3 0}}$ & $\mathbf{0 . 5 4} \pm \mathbf{0 . 0 4 3 i}$ & $\mathbf{0 . 0 7 4}$ & $\mathbf{0 . 9 2}$ & $\mathbf{1 2 . 2}$ \\
$250 \mu \mathrm{s}$ & $\frac{0.012 z}{z^{2}-0.97 z+0.17}$ & $0.75,0.22$ & 0.060 & 1.00 & $5.79,39.9$ \\
\hline
\end{tabular}

Note that compared with the identification results obtained for subject SI (Table I), it can be observed that the model gains are significantly reduced for subject SP while the natural frequency and damping ratio are slightly increased. The model with the highest dc gain (mean pulsewidth $200 \mu \mathrm{s}$ ) was selected as the nominal model for the controller design.

For the standing experiments, the inner-loop controller was designed using the pole-placement approach with the same design parameters as for subject SI (closed-loop rise-time of $0.2 \mathrm{~s}$, observer rise-time of $0.1 \mathrm{~s}$ ). For the outer-loop, both the closed-loop rise-time and the observer rise-time equal $0.7 \mathrm{~s}$. This is slightly faster than the parameters used for subject SI.

Results of a quiet standing experiment are shown in Fig. 7. The top plot shows the stimulation pulsewidth $p$, the second plot depicts the total ankle moments (both reference $m_{\text {ref }}$ and measured $m$ ) and the third plot shows the body angle (both reference $\theta_{\text {ref }}$ and measured angle, $\theta$ ). The bottom plot shows the estimated disturbance moment, $m_{\text {dist }}$, which was calculated using (3). In this experiment, the subject stands with a constant inclination angle until fatigue causes the stimulation pulsewidth to saturate and the subject to lose stability. We observed that external disturbances applied during the test would easily destabilize the subject: the subject was pulled forward at $90 \mathrm{~s}$ and pushed backwards at $110 \mathrm{~s}$. In both cases, intervention by the experimenter was required to recover stability. The maximal period of quiet standing achieved in these experiments was approximately $7 \mathrm{~min}$ (albeit with a modified controller design based on the $\mathrm{H}_{\infty}$ approach mentioned in Section II-B2[19]).

Figs. 8 and 9 show results where the subject stands quietly with a constant reference angle while a disturbance is applied.
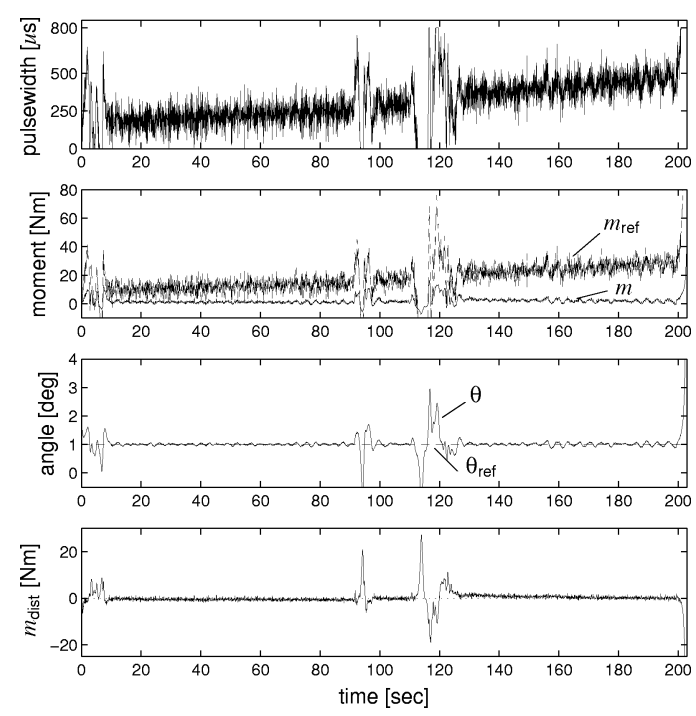

(a)
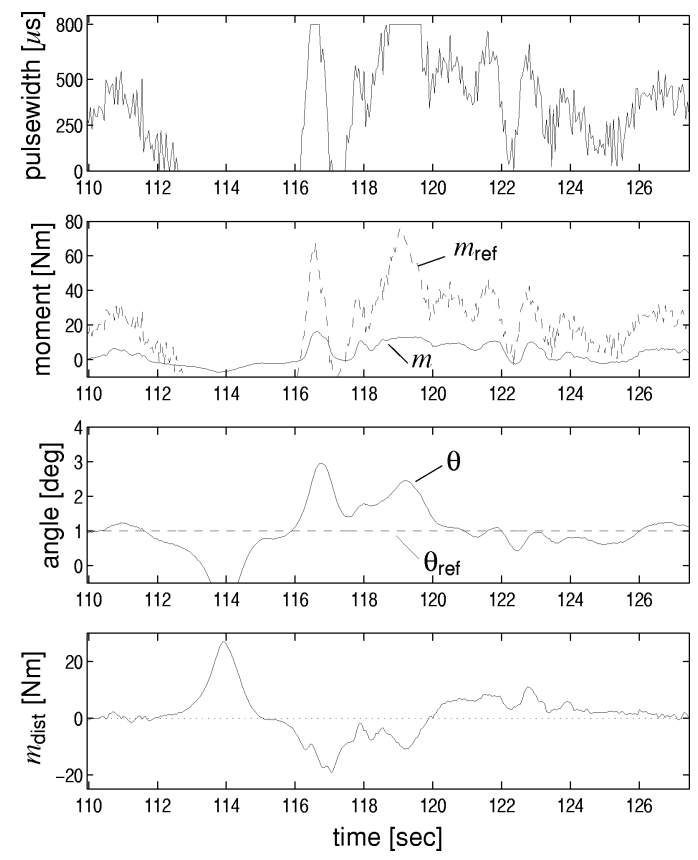

(b)

Fig. 7. Quiet standing, subject SP. The outer-loop controller is designed using pole-placement. Solid lines indicate measured values and dashed lines are reference values. (a) Full standing experiment. (b) Extract from (a): second disturbance.

In Fig. 8, the experimenter pulls anteriorly at chest level with a moment of approximately $6 \mathrm{Nm}$. The disturbance is applied at 5 and $25 \mathrm{~s}$, each time for a period of $10 \mathrm{~s}$, emulating the effect of slowly lifting a light object. This causes the subject to move forward slightly, but he is stabilized by an increase of the stimulation and the effect of the disturbance is compensated for.

In Fig. 9, the subject lifts a weight $(2.5 \mathrm{~kg})$ with his arms fully extended, from 4 to $20 \mathrm{~s}$. This is successfully compensated by an increase of the stimulation and stability is ensured. However, when he lifts the same weight for a second time (starting at $24 \mathrm{~s}$ ) the stimulation saturates quickly and the subject consequently loses balance. 

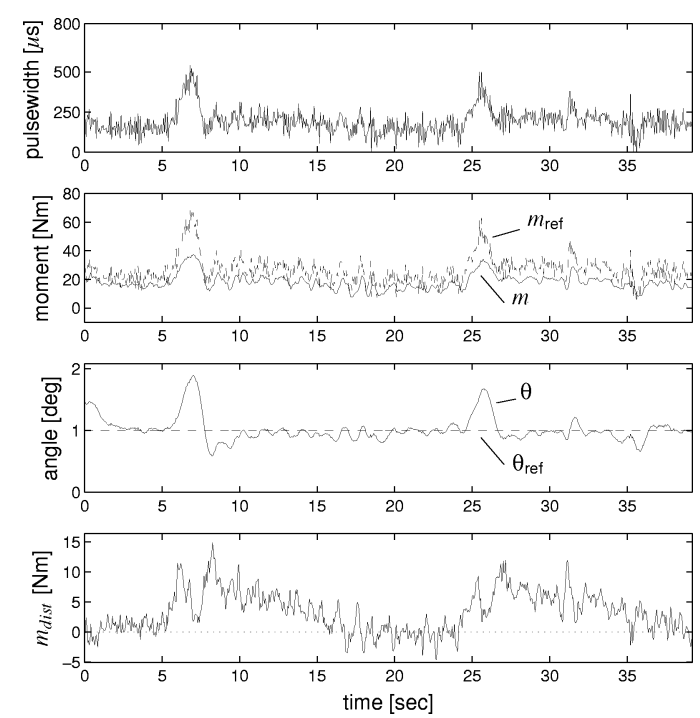

(a)
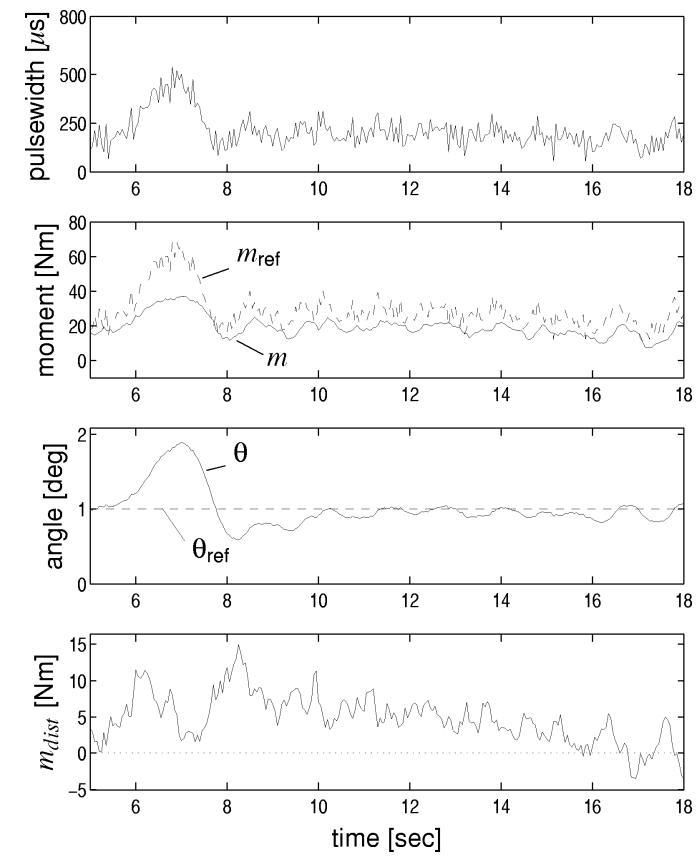

(b)

Fig. 8. Disturbed standing, subject SP. See text for details. Solid lines indicate measured values and dashed lines are reference values. (a) Full standing experiment. (b) Extract from (a): first disturbance.

Fig. 10 shows results where the reference angle changes. Although a relatively large overshoot can be observed in the measured angle when the reference angle increases (i.e., when a more forward-leaning posture is commanded), stability is achieved during the entire experiment.

\section{DISCUSSION}

The results of this study show that with our control strategy both an intact and a paraplegic subject can be stabilized in a quiet standing position for significant periods of time by electrical stimulation of the plantarflexor muscles. The stimulation level is adjusted by a feedback control structure which uses measurements of the ankle moments and of the subject's inclination angle. External disturbances can be rejected.
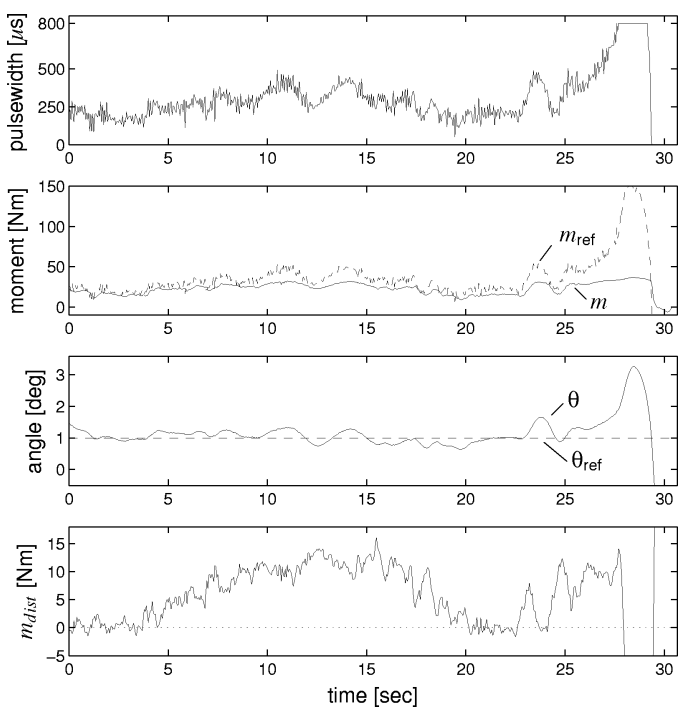

Fig. 9. Disturbed standing, subject SP. See text for details. Solid lines indicate measured values and dashed lines are reference values.
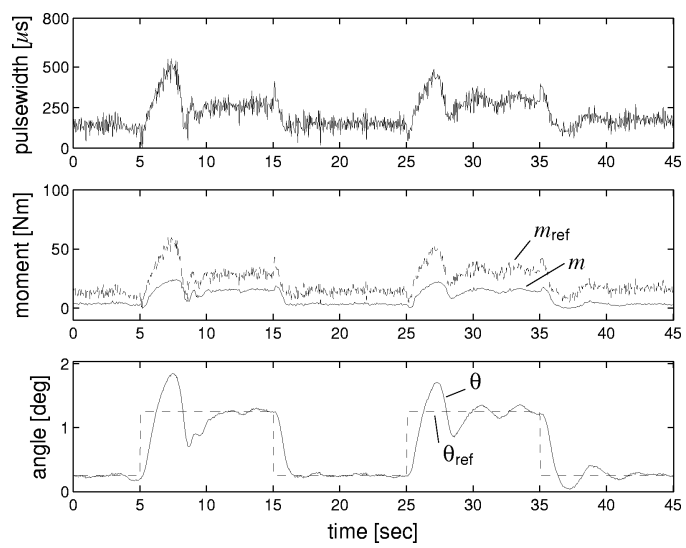

Fig. 10. Changing reference, subject SP. See text for details. Solid lines indicate measured values and dashed lines are reference values. No external disturbances were applied.

The design of the inner-loop moment controller is based on a second-order linear model of the muscle dynamics which is derived from experimental data. The identification procedure for this model requires typically only $10 \mathrm{~s}$ of stimulation for three different stimulation levels. Thus, muscle fatigue prior to standing experiments can be minimized. The muscle identification results show correspondence of the models with expected muscle properties, such as slower muscle dynamics and saturating gain with increased stimulation level. Selecting the model with the highest gain as the nominal model for the controller design yields robust moment control results, ensuring that the inner loop remains stable for other levels of stimulation at which the gain is smaller than that of the nominal model.

Using the pole-placement controller approach makes the design procedure simple, quick, and reliable. Stable standing can be achieved consistently with the same set of design parameters at different sessions, although the muscle characteristics can vary considerably from session to session. Design parameters for subject SI vary only slightly from those for subject SP, the only difference being a smaller rise-time in the outer loop for subject SP. A faster outer loop design is required for subject 
SP since the influence of fatigue is greater in this subject and a faster design provides extra stability margin when the gain decreases.

Standing results obtained for the neurologically-intact subject (Fig. 5) show that the feedback control approach performs satisfactorily. Significant external disturbances can be rejected and changes of the reference inclination angle can be applied. Progressive muscle fatigue was not observed with subject SI, and standing could be achieved for long periods of time.

For the paraplegic subject, the periods of standing which could be achieved in this study were significantly longer than those reported previously [5]. The limiting factor was progressive muscle fatigue which can, for example, be observed in the test shown in Fig. 7. We found that after the muscles were fatigued no further standing was possible even after resting periods of up to $20 \mathrm{~min}$.

The disturbance rejection tests for subject SP show that disturbances of up to $10-15 \mathrm{Nm}$ can be rejected, cf. Figs. 8 and 9. This is significantly smaller than for subject SI where disturbances of more than $20 \mathrm{Nm}$ were reliably rejected. The ability to reject disturbances is further limited by fatigue which is illustrated by the result shown in Fig. 9. Here, the subject remains stable during the first disturbance which peaks at approximately $15 \mathrm{Nm}$. A second disturbance of similar kind causes loss of balance at only $10 \mathrm{Nm}$. Comparing the stimulation pulsewidth in the top graph in Fig. 9 for the first and the second disturbance shows that a much larger pulsewidth is necessary for the second instance, indicating progressive muscle fatigue.

Results of reference tracking (Fig. 10 for subject SP) show that a given trajectory can be followed reliably and that stability can be ensured for small inclination angles.

It should be noted that the robust feedback controller design method used here is distinct from adaptive controller approaches as suggested, for example, in [22]. Adaptive control requires persistent excitation of the plant to detect changes in the plant characteristics. Since in a quiet standing (balancing) situation, disturbances and, thus, excitation of the plant can be very small for significant periods of time, adaptive control is not suitable. In this situation, the use of a single, time-invariant controller which is designed to perform robustly for the expected operating conditions is more appropriate.

The limitations of the ankle moment control in paraplegia discussed earlier underline the need to include upper body movement to support balance in practical functional arm-free standing. Matjačić and Bajd have carried out a study on unsupported standing in which the upper body is free to move [23], [24]. The results presented here can serve as a basis for the design of artificial lower-limb controllers for such systems. First experimental results of unsupported standing with integrated voluntary upper-body movement and FES-based ankle stiffness control are reported by Hunt et al. [25] and Jaime et al. [26], [27]. In a generalized setup, the body can be assumed to be a double-link inverted pendulum where the upper link is the trunk which is under voluntary control at the hip-joint. The lower link is formed by the legs and can rotate at the ankle joint which is controlled by artificial stimulation, while the knees are locked, either by mechanical bracing or by electrical stimulation.

\section{CONCLUSION}

A reliable controller design approach, based on pole-placement design, was developed and implemented which performed very consistently and robustly under varying conditions. The experience gained with this approach can potentially lead to the development of robust feedback controllers whose set up requires only minimal input by the user.

The standing results show that, while the neurologically intact subject was able to produce a significant moment at the ankle, only a relatively small ankle moment could be generated by the paraplegic subject. While this moment was sufficient to stabilize the subject for small inclination angles, disturbances could easily cause loss of balance. Although it is conceivable that larger moments at the ankle could be achieved after a more intense training regime (e.g., by training the muscles against a load), the principal limitation remains that paralyzed muscle will be significantly weaker and fatigue faster than muscles of neurologically intact subjects. Thus, while unsupported standing based on ankle moment control alone is in principle possible for paraplegic subjects, its limitations are determined by available muscle force and fatigue. The stability robustness will be further reduced by spasticity which acts as an additional external disturbance and can generally be present in paraplegic subjects.

This underlines that to support balance in practical arm-free standing system it is necessary to integrate voluntary control of the upper body with artificial control of the lower limbs where the artificial control methods for the ankle joints can be based on the results presented here. While these artificial control approaches currently require measurements of the ankle moment and inclination angle, it may be possible to reduce the need for expensive sensors by using stability analysis approaches which are based on the evaluation of the subject's center of pressure [28]. This can eventually lead to practical systems for arm-free standing in paraplegia.

\section{ACKNOWLEDGMENT}

The authors would like to thank Dr. N. Donaldson (University College London) for his contributions in the initial experiments, and C. McFadden (Salisbury District Hospital) and R.-P. Jaime (University of Glasgow) for their assistance during the experiments.

\section{REFERENCES}

[1] A. R. Kralj and T. Bajd, Functional Electrical Stimulation: Standing and Walking After Spinal Cord Injury. Boca Raton, FL: CRC, 1989.

[2] L. A. Benton, L. L. Baker, B. R. Bowman, and R. L. Waters, "Functional electrical stimulation-A practical clinical guide," Rancho Los Amigos Rehab. Eng. Center, Downey, CA, 1981.

[3] N. Donaldson and C.-H. Yu, "A strategy used by paraplegics to stand up using FES,” IEEE Trans. Rehab. Eng., vol. 6, pp. 162-167, June 1998.

[4] - "FES standing: Control by handle reactions of leg muscle stimulation (CHRELMS)," IEEE Trans. Rehab. Eng., vol. 4, pp. 280-284, Dec. 1996.

[5] K. J. Hunt, M. Munih, and N. Donaldson, "Feedback control of unsupported standing in paraplegia. Part I: Optimal control approach. Part II: Experimental results," Trans. IEEE Rehab. Eng., vol. 5, pp. 331-352, Dec. 1997. 
[6] D. E. Wood, V. Harper, F. M. D. Barr, P. N. Taylor, G. F. Phillips, and D. J. Erwins, "Experience in using knee angles as part of a closed loop algorithm to control FES assisted paraplegic standing," in Proc. 6th Int. Workshop FES, Vienna, Austria, Sept. 1998, pp. 137-140.

[7] D. Graupe and K. H. Kohn, Functional Electrical Stimulation for Ambulation by Paraplegics: Twelve Years of Clinical Observations and System Studies. Melbourne, FL: Krieger, 1994.

[8] G. Khang and F. E. Zajac, "Paraplegic standing controlled by functional neuromuscular stimulation: Part I-Computer model and control-system design. Part II-Computer simulation studies," IEEE Trans. Biomed. Eng., vol. 36, pp. 873-894, Sept. 1989.

[9] R. J. Jaeger, "Design and simulation of closed-loop electrical stimulation orthoses for restoration of quiet standing in paraplegia," J. Biomech., vol. 19 , no. 10 , pp. $825-835,1986$.

[10] N. Donaldson, "Practical ankle controllers for unsupported standing in paraplegia," in Proc. Ljubljana FES Conf., 1993, pp. 61-64.

[11] N. Donaldson, F. M. D. Barr, G. F. Phillips, and T. A. Perkins, "Unsupported standing of paraplegics by stimulation of the plantarflexors: Some results from the Wobbler apparatus," in Neuroprosthetics: From Basic Research to Clinical Application, A. Pedotti, M. Ferrarin, J. Quintern, and R. Riener, Eds. Berlin, Germany: Springer-Verlag, 1996, pp. 217-232.

[12] N. Donaldson, M. Munih, G. F. Phillips, and T. A. Perkins, "Apparatus and methods for studying artificial feedback-control of the plantarflexors in paraplegics without interference from the brain," Med. Eng. Phys., vol. 19, no. 6, pp. 525-535, 1997.

[13] K. J. Hunt, H. Gollee, R. Jaime, and N. Donaldson, "Design of feedback controllers for paraplegic standing," in Proc. IEE Control Theory and Applications, vol. 148, Mar. 2001, pp. 97-108.

[14] K. J. Hunt, H. Gollee, R.-P. Jaime, and N. Donaldson, "Feedback control of unsupported standing," Technol. Health Care, vol. 7, no. 6, pp. 443-447, 1999.

[15] L. Ljung, System Identification: Theory for the User, 2nd ed. Englewood Cliffs, NJ: Prentice-Hall, 1999.

[16] K. J. Åström and B. Wittenmark, Computer-Controlled Systems. Theory and Design, 3rd ed. Englewood Cliffs, NJ: Prentice-Hall, 1997.

[17] G. F. Franklin, J. D. Powell, and A. Emami-Naeini, Feedback Control of Dynamic Systems, 3rd ed. Reading, MA: Addison-Wesley, 1994.

[18] W. Holderbaum, K. Hunt, and H. Gollee, "Application of $\mathrm{H}_{\infty}$ robust control to paraplegic standing," in Proc. 15th IFAC World Congr., Barcelona, Spain, July 2002, p. 208.

[19] W. Holderbaum, K. J. Hunt, and H. Gollee, " $H_{\infty}$ robust control design for unsupported paraplegic standing: Experimental evaluation," Contr. Eng. Practice, vol. 10, no. 11, pp. 1211-1222, 2002.

[20] G. F. Phillips, J. R. Adler, and S. J. G. Taylor, "A portable programmable eight-channel surface stimulator," in Proc. Ljubljana FES Conf,, 1993, pp. $166-168$

[21] K. J. Hunt, M. Munih, N. D. Donaldson, and F. M. D. Barr, "Investigation of the Hammerstein hypothesis in the modeling of electrically stimulated muscle," IEEE Trans. Biomed. Eng., vol. 45, pp. 998-1009, Aug. 1998.

[22] J. J. Abbas and J. C. Gillette, "Using electrical stimulation to control standing posture," IEEE Control Syst. Mag., vol. 21, pp. 80-90, Aug. 2001.

[23] Z. Matjačić and T. Bajd, "Arm-free paraplegic standing. Part I-Control model synthesis and simulation. Part II-Experimental results," IEEE Trans. Rehab. Eng., vol. 6, pp. 125-150, June 1998.

[24] Z. Matjačić, "Control of ankle and hip joint stiffness for arm-free standing in paraplegia," Neuromodulation, vol. 4, no. 1, pp. 37-46, 2001.

[25] K. J. Hunt, H. Gollee, and R.-P. Jaime, "Control of paraplegic ankle joint stiffness using FES while standing," Med. Eng. Phys., vol. 23, no. 8, pp. 541-555, 2001

[26] R.-P. Jaime, Z. Matjačić, and K. J. Hunt, "Paraplegic standing supported by FES-controlled ankle stiffness," in Proc. 6th Annu. Conf. Int. FES Soc., Cleveland, OH, 2001, pp. 68-70.
[27] _ - "Paraplegic standing supported by FES-controlled ankle stiffness," IEEE Trans. Neural Sys. Rehab. Eng., vol. 10, pp. 239-248, Dec. 2002

[28] M. R. Popović, I. P. I. Pappas, K. Nakazawa, T. Keller, M. Morari, and V. Dietz, "Stability criterion for controlling standing in able-bodied subjects," J. Biomech., vol. 33, no. 11, pp. 1359-1368, 2000.

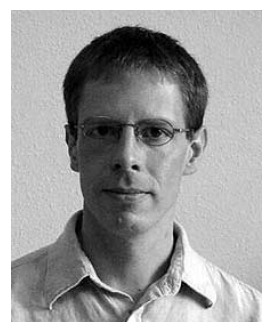

Henrik Gollee (M'00) received the Dipl.-Ing. degree in electrical engineering from the Technical University of Berlin, Berlin, Germany, in 1995 and the Ph.D. degree in systems and control from the University of Glasgow, Glasgow, Scotland, U.K., in 1998.

$\mathrm{He}$ is currently a Lecturer with the Department of Mechanical Engineering and the Assistant Director of the Center for Rehabilitation Engineering, Glasgow University, Glasgow. He has been involved with collaborative research projects with the National Spinal Injuries Unit, Glasgow, include applications of FES in balance control, cycling, arm-cranking exercise, and respiratory support. His research interests include the application of advanced control methods in assistive technology and human-machine interface, with the primary focus on applications in spinal-cord injury rehabilitation.

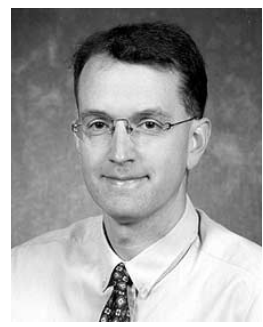

Ken J. Hunt (S'87-M'87) received the B.Sc. degree in electrical and electronic engineering (first-class honors) and the Ph.D. degree in control theory from the University of Strathclyde, Glasgow, Scotland, U.K., in 1984 and 1987, respectively.

From 1989 to 1992 , he held a Royal Society of Edinburgh Personal Research Fellowship, and from 1992 to 1997, he was a Research Scientist and Project Leader with Daimler-Benz Systems Technology Research, Berlin, Germany. He is currently a Wylie Professor of Mechanical Engineering and the Director of the Center for Rehabilitation Engineering, Glasgow University, Glasgow. He is also a Clinical Research Professor with the National Spinal Injuries Unit, Southern General Hospital, Glasgow. His research interests include feedback control of balance in paraplegia, lower-limb cycling in paraplegia, and assisted upper-limb arm-cranking exercise in tetraplegia.

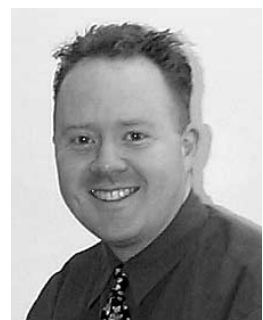

Duncan E. Wood received the B.Eng. degree in electronic engineering from the University of Southampton, Southampton, U.K., in 1989, and the M.Sc. and Ph.D. degrees in biomedical engineering from the University of Surrey, Surrey, U.K. in 1990 and 1994, respectively.

Since 1995, he has worked at the Salisbury District Hospital, Salisbury, U.K., as a Clinical Scientist in a multidisciplinary team on functional electrical stimulation programs to correct neurological disorders. He is also a Senior Lecturer with Bournemouth University, Bournemouth, U.K., within the Academic Biomedical Engineering Research Group. His research interests include using electrical stimulation (surface or implanted) to restore lower limb functions, biomechanics and control of standing, analysis of and characterization of gait and muscle activity, quantifying measurements of spasticity, and the development of algorithms to control stimulation. 